Check for updates

Cite this: RSC Adv., 2017, 7, 36605

\title{
Exploitation of mussel byssus mariculture waste as a water remediation material $\dagger$
}

\author{
Devis Montroni, ${ }^{a}$ Corrado Piccinetti, ${ }^{b}$ Simona Fermani, ${ }^{a}$ Matteo Calvaresi, (D) ${ }^{a}$ \\ Matthew J. Harrington ${ }^{* c}$ and Giuseppe Falini (D) *a
}

Dye pollution represents an important environmental concern, especially from textile industries in the South of Asia. On the other hand, biomass accumulation derived from mussel processing in alimentary industries is also an environmental problem. In this research, these two environmental issues are addressed by proposing the reuse of mussel's protein anchoring threads, the byssus, as disposable material for dye water removal. The byssus was selected as substrate because it contains a distinctive variety of functional groups that can be exploited for diverse chemical interactions. This material was utilized in its native metaled state and in the de-metaled one, in order to study how the chemical state of the functional groups influences the adsorption properties of both anionic and cationic aromatic dyes. The results of comparative experiments of adsorption showed a higher uptake in the native metaled byssus for a model cationic dye, methylene blue, while the de-metaled byssus showed a higher uptake for a model anionic dye, Eosin Y. These results are considered in light of different uptake mechanisms, supported by the analyses of isotherm model parameters, in which diverse functional groups are involved as a function of substrate and dye chemical states.

Received 14th June 2017 Accepted 19th July 2017

DOI: $10.1039 / c 7 r a 06664 c$

rsc.li/rsc-advances year of solid residues, which are generally discarded. ${ }^{6,7}$ They are constituted mainly of shells and minor amounts of soft tissue and byssus. Many researchers have investigated how to recover these solid residues. ${ }^{8}$ Most work has utilized the shell to remedy to phosphorus or fluoride pollution, ${ }^{9,10}$ as a substrate for heterogeneous catalysts ${ }^{11,12}$ or to produce hydroxyapatite; $;^{13}$ while very few studies focused on the recovery of byssus. ${ }^{14,15}$ The byssus is a protein-based fibrous holdfast utilized by mussels to anchor to different substrates in marine environments and avoid being dislodged by currents and waves. ${ }^{16-18}$

The aim of this research is to investigate the use of byssus as an adsorbent matrix for water dye removal. The application of adsorption procedures has become very popular among wastewater treatments due to its effectiveness, low cost, easy design and simplicity in use and maintenance. ${ }^{2,19}$ One of the main problems associated with the use of adsorption matrices is their regeneration, ${ }^{20}$ leading current research efforts towards generating disposable substrates. This approach has been used by employing different matrix such as almond shell, ${ }^{21}$ banana peel, ${ }^{22}$ rice, ${ }^{23,24}$ and chitosan, ${ }^{25}$ as well as plenty of other examples. ${ }^{26}$

The choice of byssus is justified, beside the above considerations, by the knowledge that its structure contains a high number of potential binding sites for dyes based on its unique structure and chemistry. The byssus ultra-structure organization is generally split in four regions (Fig. 1) having different structure and chemical composition, namely: (i) the plaque; (ii) the thread core; (iii) the thread cuticle and (iv) the stem. ${ }^{27}$ The plaque anchors the substrate and is composed of proteins rich in dihydroxyphenylalanine (DOPA) residues, ${ }^{28}$ which coordinate $\mathrm{Fe}^{3+}$
aDipartimento di Chimica "Giacomo Ciamician”, Alma Mater Studiorum Università di
Bologna, via Selmi 2, 40126 Bologna, Italy. E-mail: giuseppe.falini@unibo.it
${ }^{b}$ Laboratory of Fisheries and Marine Biology, University of Bologna, viale Adriatico 1)
N, I-61032, Fano, PU, Italy
'Department of Biomaterials, Max-Planck Institute for Colloids and Interfaces,
Research Campus Golm, Potsdam 14424, Germany. E-mail: Matt.Harrington@
mpikg.mpg.de
$\dagger$ Electronic supplementary information (ESI) available: Adsorption kinetics;
adsorption isotherm models. See DOI: 10.1039/c7ra06664c 


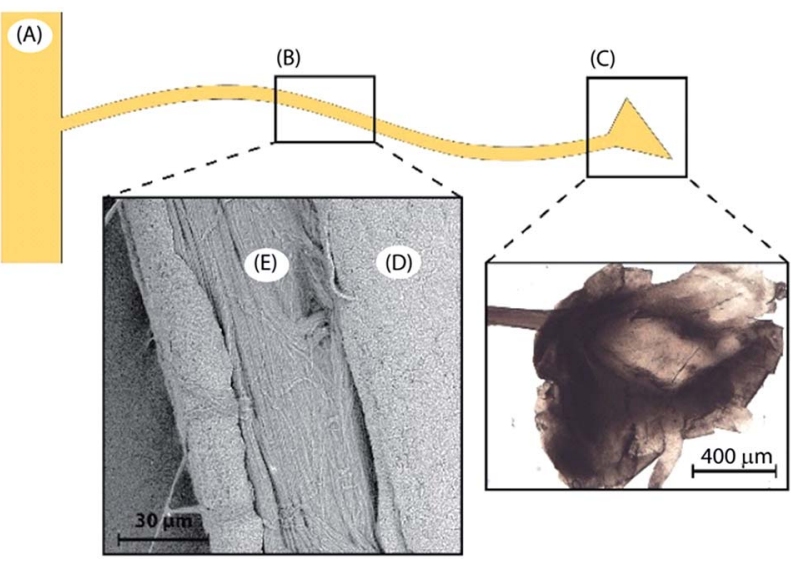

Fig. 1 Byssus different regions: (A) the stem, (B) the thread and (C) the plaque. The thread itself is also divided in two more regions, (D) the external cuticle and $(E)$ the inner fibrous core.

in very stable complexes. ${ }^{28}$ The thread cuticle also contains DOPArich proteins, which bind to $\mathrm{Fe}^{3+}$ to create a self-healing material. ${ }^{29}$ Notably, polymers having poly-DOPA regions have been used for water remediation. ${ }^{30}$ The thread core is mainly composed of proteins called preCols, ${ }^{31}$ which are collagen-based proteins having terminal histidine (His)-rich domains binding $\mathrm{Cu}^{2+}$ and $\mathrm{Zn}^{2+}{ }^{32}$ The stem connects the single byssal thread to the organism and has a composition similar to that of the thread. It is worth noting that collagen based matrices have been tested for water remediation from anionic dyes, ${ }^{33,34}$ while the use of poly-His in water remediation has been utilized specifically for metal ion removal. ${ }^{35}$ Thus, the ability of byssus to participate in a wide range of different chemical interactions due to co-presence of DOPA-rich and Hisrich proteins, together with collagen, may provide the potential for binding a range of different dyes for water remediation. To test the capability of byssus threads as a bio-renewable adsorption matrix, we tested this material on Methylene Blue (MB) as a test molecule for cationic dyes, and on Eosin Y (EosY), as test molecule for anionic dyes. These test molecules were chosen because of their easy detectability using UV-Vis spectroscopy and their low toxicity. In this work both the native byssus, with its native metal ion content and a de-metaled byssus were used. This comparison was done to understand how the diverse coordination state of the metal binding functional groups affects the performance of this matrix in the removal of dye from wastewater.

\section{Materials and methods}

\section{Materials}

Reagents and solvents were purchased from Sigma Aldrich and utilized without any further purification. EosY and MB daily fresh solutions were prepared for each experiment. The byssus was collected from a mussel-breeding farm close to Fano (Italy).

\section{Byssus pre-treatment}

The byssus was mechanically collected from the mussels by hand and rinsed with water and detergent until the washing
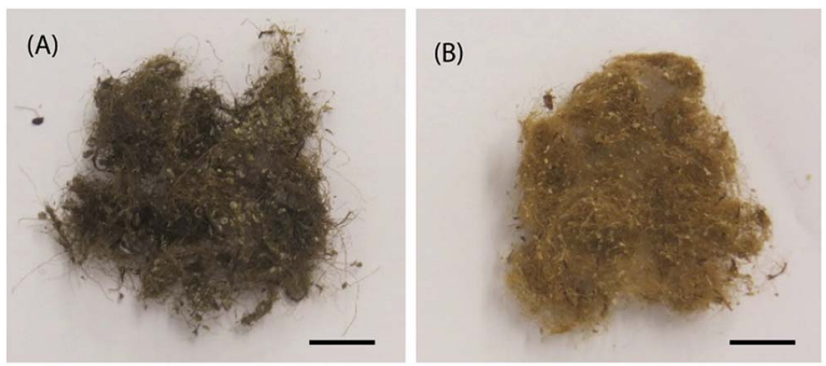

Fig. 2 Photographs of the native metaled byssus (A) and of the demetaled one (B). The colour of the material change according to the metal content. In the presence of $\mathrm{Fe}^{3+}$ the byssus appears brownblack, while when it is almost metal free its colour is gold-like. Scale bar of $10 \mathrm{~mm}$.

water was completely clear. Afterwards, it was washed again with de-ionized water to remove the detergent, stirred twice in ethanol for 30 minutes and finally washed again twice with deionized water for 15 minutes to rehydrate the threads and remove the ethanol. The clean air-dried byssus was stored in a desiccator under vacuum (Fig. 2A).

\section{Byssus de-metallation}

The de-metallation process was carried out following the procedure reported by Schmitt et al. ${ }^{36}$ in which EDTA was used as a chelator. The protocol was modified using $0.1 \mathrm{M}$ Tris buffer and adding two more $1 \mathrm{~h}$ wash in milliQ water at the end of the procedure to ensure full removal of EDTA. The airdried sample was conserved in a desiccator under vacuum (Fig. 2B).

\section{Optical microscope images}

Optical microscope images were collected using a SM-LUX POL microscope and a Leica EC3 camera. When thread cross sections were analyzed $40 \mu \mathrm{m}$ samples were cut using a Cryomicrotome (Cryostat Microm HM560).

\section{Dye removal experiments}

Dye removal experiments were carried out inserting $50 \mathrm{mg}$ of byssus in $1.5 \mathrm{~mL}$ of solution having different concentrations of dye. The byssus incubation time was of 48 and $72 \mathrm{~h}$ for MB and EosY solutions, respectively. The dye concentration in solution was measured before and after the byssus insertion by using a UV-Vis spectrophotometer (Cary60, Agilent Technologies) using a spectral range of $600-810 \mathrm{~nm}$ for $\mathrm{MB}$ and 450-810 for EosY. The MB and EosY starting concentrations used were $0.01,0.1,0.5,1,2$ and $5 \mathrm{mM}$. Each incubation experiment was carried out in double at $22{ }^{\circ} \mathrm{C}$. The $5 \mathrm{mM}$ starting solution of EosY and $\mathrm{MB}$ had a $\mathrm{pH}$ of 7.1 and 8.2, respectively.

\section{Adsorption and desorption kinetics}

Adsorption kinetics experiments were carried out using $50 \mathrm{mg}$ of byssus in $1.5 \mathrm{~mL}$ of a $0.01 \mathrm{mM}$ solution of dye and recording 
the spectra every 10 minutes for the first 300 minutes and then every 15 minutes until $72 \mathrm{~h}$. The desorption kinetics were done using the same parameters by inserting samples from the dye removal experiments in $1.5 \mathrm{~mL}$ of Pre-milliQ water (resistivity 18.2 $\mathrm{M} \Omega \mathrm{cm}$ at $25^{\circ} \mathrm{C}$; filtered through a $0.22 \mu \mathrm{m}$ membrane) for $72 \mathrm{~h}$ at a temperature of $22^{\circ} \mathrm{C}$. All the kinetics experiments were carried out using a Cary60 from Agilent Technologies equipped with an 18-cell holder at $22^{\circ} \mathrm{C}$.
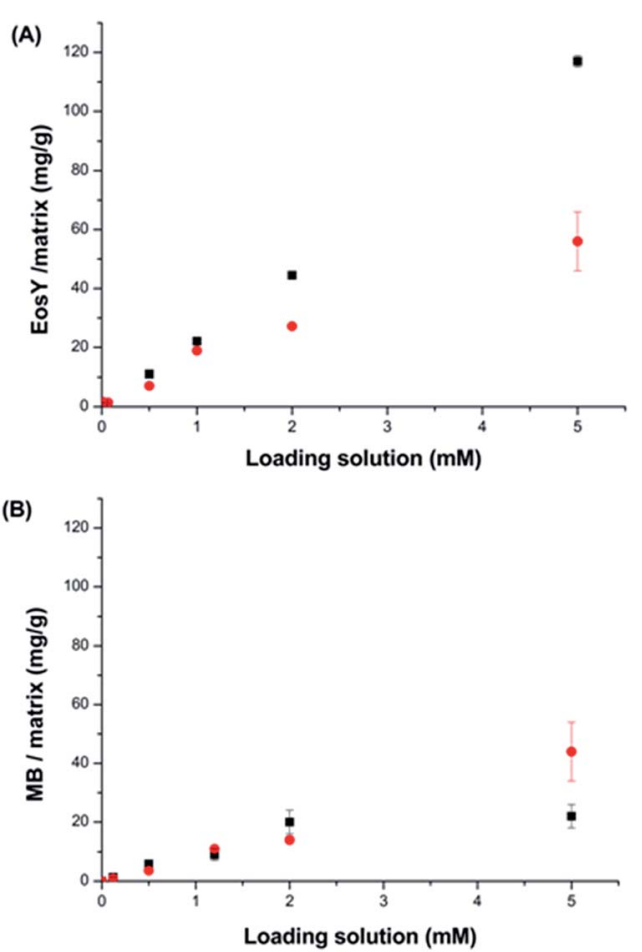

Fig. 3 Dye removal of (A) EosY from $1.5 \mathrm{~mL}$ of dye solution and (B) MB in the same experimental conditions. In red are reported the data collected using native byssus and in black those using de-metaled byssus.

\section{Results}

The adsorption kinetics of the byssus was evaluated by incubating a defined mass of byssus into a dye reference solution (0.01 mM). The kinetic data showed that the two dyes interacted differently with the native byssus. While MB was completely adsorbed from the solution in the matrix in less than $24 \mathrm{~h}$, EosY required more time and after $72 \mathrm{~h}$ was almost completely removed from the solution. The de-metaled byssus, instead, behaved differently with respect to the two dyes. When these adsorption data were compared to those on the native ones, EosY exhibited a similar kinetic of adsorption, while MB a slower one (Fig. SI1 and SI $\dagger$ ). Following the information from these preliminary experiments, $48 \mathrm{~h}$ for $\mathrm{MB}$ and $72 \mathrm{~h}$ for EosY were chosen as the incubation times for the byssus in the different dye solutions during removal experiments, assuming that these times ensured the maximum dye loading into the byssus threads, independently from the starting dye concentration.

The results of the dye removal experiments are reported in Fig. 3 and Table 1, while an optical view of experiments is shown in Fig. 4. EosY presented a higher affinity for the de-metaled byssus (Fig. 3A), while the MB appeared to have a higher affinity to the native one (Fig. 3B). The analysis of the results reported in Table 1 shows that the $50 \mathrm{mg}$ of byssal threads were able to remove completely the dye from solutions having a dye concentration below or equal to $0.5 \mathrm{mM}$. Above this concentration different trends were observed. EosY was removed from solutions more efficiently by using de-metaled byssus than the native one. Indeed, when a $5 \mathrm{mM}$ EosY solution was used, a loading of EosY equal to $117 \pm 2 \mathrm{mg} \mathrm{g}^{-1}$ and $56 \pm 10 \mathrm{mg} \mathrm{g}^{-1}$ was obtained on de-metaled and native byssus, respectively. MB was more efficiently removed from solution by the native byssus than the de-metaled one. In this case the MB loading from a $5 \mathrm{mM}$ MB solution was equal to $38 \pm 1 \mathrm{mg} \mathrm{g}^{-1}$ and $72 \pm 17 \mathrm{mg}$ $\mathrm{g}^{-1}$ on de-metaled and native byssus, respectively.

Adsorption isotherm models provide useful data in order to understand the mechanisms of the adsorption process, to describe how an adsorbate interacts with a substrate and to

Table 1 Concentration of dye in the matrix $\left(\mathrm{mg} \mathrm{g}^{-1}\right)$ with the percentage of dye adsorbed from the solution (wt\%) shown in parentheses for removal experiments starting from different loading solution using native byssus and de-metaled byssus

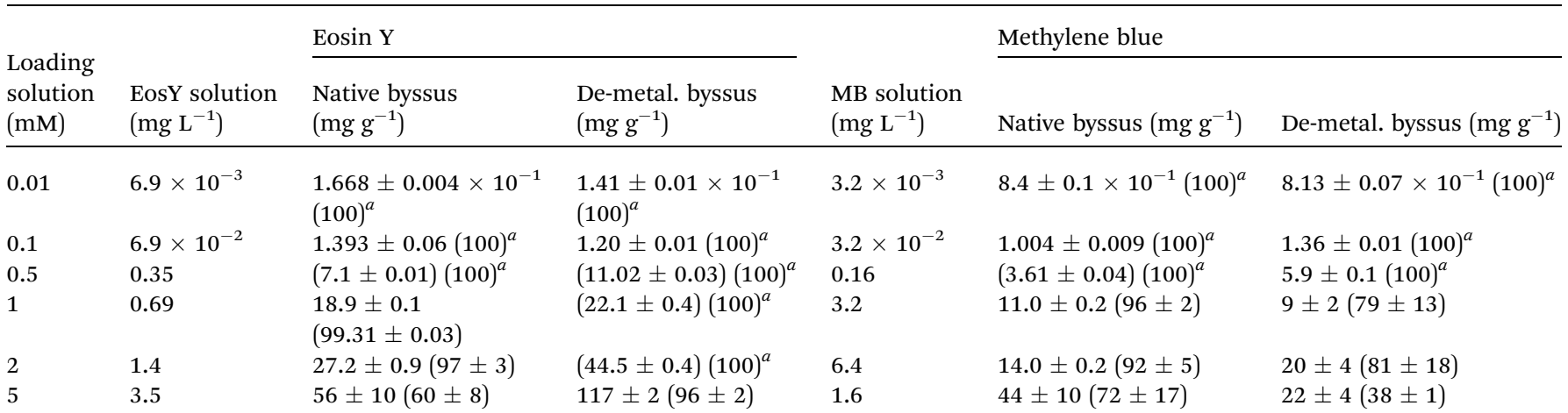

${ }^{a}$ Refers to samples with an after treatment concentration in solution lower than the detectable limit of the instrument $(2 \mu \mathrm{M}$ for EosY and $5 \mu \mathrm{M}$ for $\mathrm{MB})$, so the dye removal was assumed to be quantitative. 


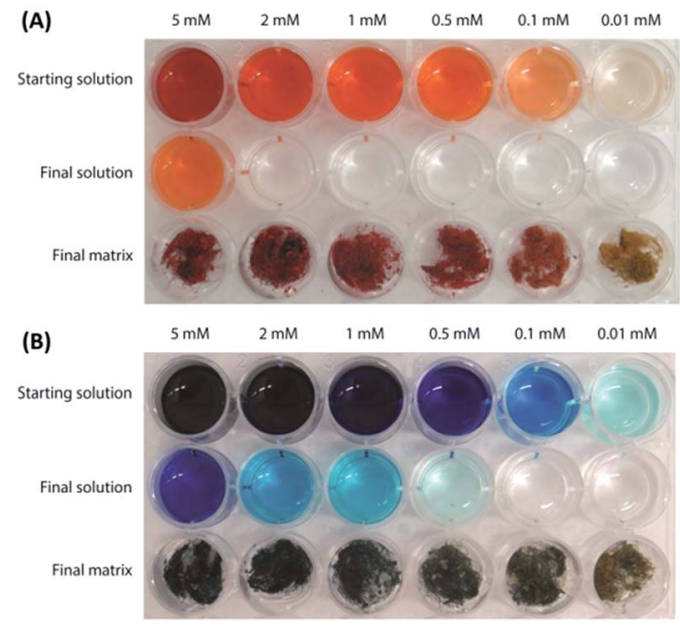

Fig. 4 Camera photographs illustrating the dye removal experiments using (A) de-metaled byssus on EosY solutions, and (B) native byssus on MB solutions.

evaluate the applicability of the process. The Langmuir, Freundlich, and Dubinin Radushkevich (D-R) isotherm models were used to describe the relationship between the amount of dye adsorbed onto the byssus thread matrices and their equilibrium concentrations in aqueous solution. The experimental equilibrium data of EosY and $\mathrm{MB}$ on native and de-metaled byssus were compared with the theoretical equilibrium data obtained from the Langmuir, Freundlich, and D-R isotherm models (Fig. SI3SI5 $\dagger$ ). The isotherm constants were calculated by evaluating the linearized form of the models (see ESI $\dagger$ ). All of the isotherm constants and correlation coefficients are given in Table 3. The adsorption capacity $\left(q_{\mathrm{m}}\right)$ of EosY was found to be $46.7 \mathrm{mg} \mathrm{g}^{-1}$ and $85.5 \mathrm{mg} \mathrm{g}^{-1}$ and that of MB was $22.6 \mathrm{mg} \mathrm{g}^{-1}$ and $16.3 \mathrm{mg} \mathrm{g}^{-1}$ on native and de-metaled byssus, respectively, by using the Langmuir model equation. The $R_{\mathrm{L}}$ values were below 1 , indicating a favorable adsorption of EosY and MB on the byssus substrates under the studied conditions. The Freundlich isotherm constant,

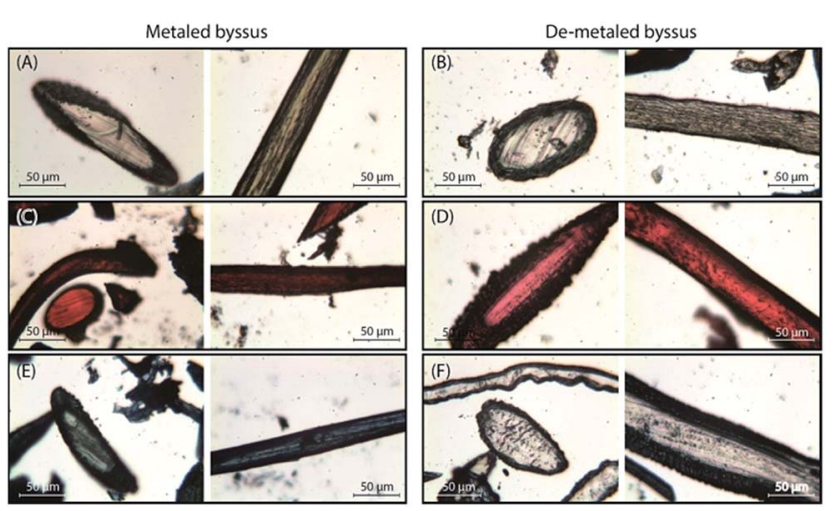

Fig. 5 Optical microscopy images of byssus thread section of $40 \mu \mathrm{m}$. On the left, metaled byssus threads, on the right, de-metaled byssus threads. (A) and (B) were control samples, (C) and (D) were treated with EosY $1 \mathrm{mM}$ and $(E)$ and $(F)$ were treated with $M B 1 \mathrm{mM}$. For each condition is present a longitudinal section of the thread and a transversal one.

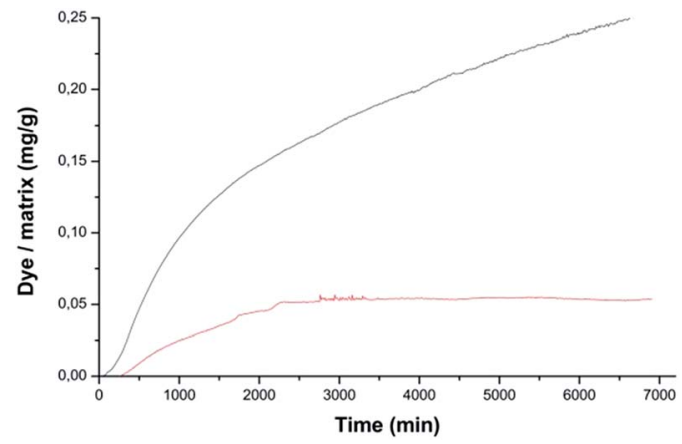

Fig. 6 Desorption kinetics of dye from previously loaded byssus samples. The dye released is reported as mass of dye $(\mathrm{mg})$, normalized over the mass of the loaded byssus thread, as a function of the time (up to $72 \mathrm{~h}$ ). The red curve reports the desorption kinetics of EosY from a native byssus, previously adsorbed with EosY $1 \mathrm{mM}$, in $1.5 \mathrm{~mL}$ of water. The black curve reports the desorption kinetics of $M B$ from a de-metaled byssus, previously adsorbed with $\mathrm{MB} 1 \mathrm{mM}$, in $1.5 \mathrm{~mL}$ of water.

$n$, gives an idea for the favorability of the adsorption process. The value of $n$ should be less than 10 and higher than unity for favorable adsorption conditions. As can be seen from Table 3, the $n$ values for EosY and MB were in the range of 3.8-5.7. By evaluating the D-R isotherm model, a rough estimation of the mean adsorption energy ( $E$, Table 3 ) values were found to be $0.21 \mathrm{~kJ}$ $\mathrm{mol}^{-1}$ and $0.79 \mathrm{~kJ} \mathrm{~mol}^{-1}$ for EosY and those for MB were $0.38 \mathrm{~kJ}$ $\mathrm{mol}^{-1}$ and $2.0 \mathrm{~kJ} \mathrm{~mol}^{-1}$ on native and de-metaled byssus, respectively. If the value of $E$ lies between 8 and $16 \mathrm{~kJ} \mathrm{~mol}^{-1}$, the adsorption process takes place chemically, whereas when $E<8 \mathrm{~kJ}$ $\mathrm{mol}^{-1}$, the adsorption process proceeds physically (see ESI $\uparrow$ ). By comparing the correlation coefficient values obtained from the isotherm models, it can be concluded that the Freundlich isotherm model is the more suitable to describe the experimental data.

Threads treated with $1 \mathrm{mM}$ solutions were cut in sections and observed using an optical microscope to investigate the dye distribution into the external cuticle and inner fibrous core of the byssus. The images (Fig. 5) showed that the dyes were homogeneously distributed in the threads in all the conditions examined except for MB uptake into de-metaled byssus. In this matrix a selective uptake of MB for cuticles was observed.

To investigate the dye desorption from the byssus, the byssal samples treated with $0.01,0.1$ and $1 \mathrm{mM}$ dye solutions were immersed in $1.5 \mathrm{~mL}$ of water. The corresponding kinetics are shown in Fig. 6, while the results are reported in Table 2. The only two conditions releasing dye after $72 \mathrm{~h}$ were $\mathrm{MB} / \mathrm{de}-$ metaled byssus and EosY/native byssus treated with $1 \mathrm{mM}$ solutions, consistently with all the previous experiments. However, for EosY, desorption was very small, while for MB desorption, it was around $6 \%$.

\section{Discussion}

The experimental results showed that the byssal threads, both in the metaled native state and in the de-metaled state, act efficiently in removing charged aromatic dyes from water. The 
Table 2 Percentage (wt\%) of dye desorbed from the matrix in $1.5 \mathrm{~mL}$ of water after $72 \mathrm{~h}$ from byssal threads dye loaded using different starting concentrations of dye

\begin{tabular}{|c|c|c|c|c|}
\hline Loading solution (mM) & Native byssus (wt\%) & De-met. byssus (wt\%) & $\begin{array}{l}\text { Native byssus } \\
\text { (wt\%) }\end{array}$ & De-metal. byssus (wt\%) \\
\hline 0.01 & $-^{a}$ & - & - & - \\
\hline 0.1 & - & - & - & - \\
\hline 1 & $0.18 \pm 0.01$ & - & - & $6 \pm 1$ \\
\hline
\end{tabular}

loading capacity of these materials, up to more than $10 \mathrm{wt} \%$ of the starting mass, are of the same order of magnitude of other waste materials. ${ }^{17-22}$ In addition, it has to be considered that from mussel mariculture wastes, ${ }^{6,7}$ with a potential capability of dye removal of about 0.5 ktones per year. This value represents about $50 \mathrm{wt} \%$ of the of dye pollution per year in waste waters from textile sector. ${ }^{2}$ In addition the byssal threads offer the possibility to be tunable with respect positive or negative charged aromatic dyes. This can be done controlling the metal ions content in the byssus threads via chelation treatment with EDTA, which may reflect the diverse possible chemical interactions by the DOPA and His residues in the byssus proteins. Also the $\mathrm{pH}$ is a parameter that could possibly be used to control the adsorption capability of byssus, since the functional groups, which are likely responsible for interaction between dye molecules and adsorbent, can be protonated or deprotonated to produce different surface charges in solution having different $\mathrm{pH}$ values. This parameter has not been investigated in the present research. However, it has been proved (personal information) that the byssus is stable in a range of $\mathrm{pH}$ from 2 to 10 . It can be considered that at acidic $\mathrm{pH}$ values the histidine groups of byssus become protonated and this changes their uptake capability of charged dyes. On the other hand, basic $\mathrm{pH}$ values induce the deprotonation of cuticle localized DOPA residues, affecting the dye uptake for both a change in the interactions and a potential shielding effect of the negatively charged cuticle. In addition to these general considerations, the byssus in acidic environment will bind metal ions less tightly. In this study, the experiments were carried out at ambient temperature. The byssus is a material that is very stable with the temperature, experimental data indicate that its structure remains unaffected up to a temperature of at least $80{ }^{\circ} \mathrm{C}$ (personal communication). Thus, it can be reasonably supposed that byssus adsorption capability should not be drastically affected by the temperature.

Table 3 Langmuir, Freundlich and Dubinin Radushkevich (D-R) isotherm parameters for the adsorption of MB and Eos $Y$ on byssus threads (see ESI)

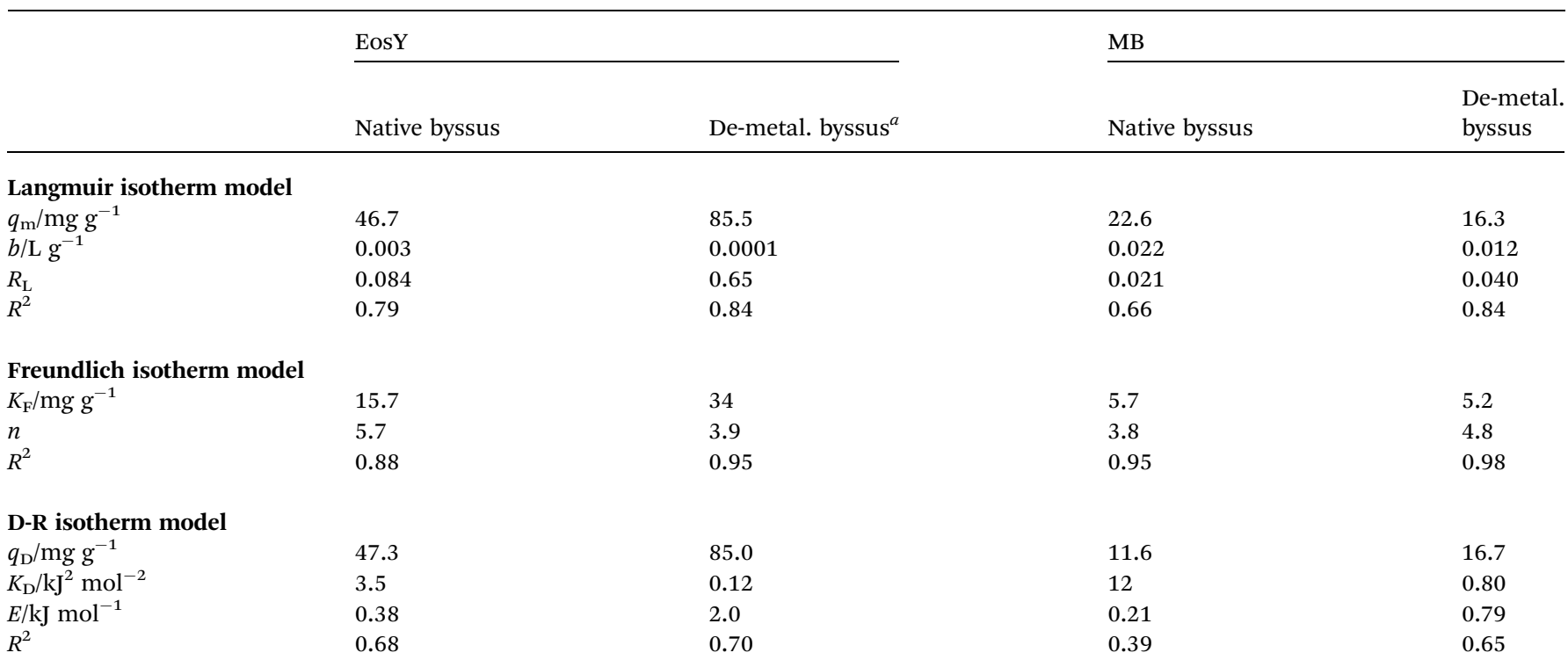
${ }^{a}$ This substrate shows a high adsorption capacity for EosY that prevents, for low starting concentrations, an accurate evaluation of the equilibrium
concentrations. 
The two dyes used represents model molecules that differ almost exclusively for the ionic charge. The native byssus showed a high affinity for a cationic dye, i.e. $\mathrm{MB}$, as illustrated by the adsorption kinetics, which showed that the dye was completely absorbed in less than $24 \mathrm{~h}$. Furthermore, the experiments of dye removal from solutions exhibited an almost complete removal of the dye up to a starting concentration of $2 \mathrm{mM}$, with EosY showing similar behavior in loading.

The desorption kinetics confirmed this affinity data, showing no desorption for MB and only a very low desorption of EosY. In other words, the dyes bind quite strongly to the byssus material. Both dyes appeared to be absorbed homogeneously along the different parts of the byssus suggesting no selective interactions with the diverse molecules and functional groups of the native byssus. De-metaled byssus, on the other hand, appeared to prefer anionic dyes, i.e. EosY. As for the native one, the dye removal experiments showed an almost complete removal of EosY in all the concentrations studied. This is different from $\mathrm{MB}$, which showed saturation in the dye uptake for concentrations higher than $0.5 \mathrm{mM}$.

These considerations are supported by the adsorption isotherm model results, which show that the best experimental data fitting occurs using the Freundlich isotherm model. This model assumes that the adsorption takes place on heterogeneous surface sites, which have different adsorption energies. The analysis of mean adsorption energy values (from the D-R isotherm model) may suggest physical interactions. This fits with wide variety of interactions offered by the byssus structural complexity and richness of molecular moieties and functional groups.

The desorption kinetics were in line with the uptake data and, while EosY was not desorbed, MB was desorbed when loaded from a $1 \mathrm{mM}$ solution. This last result was different from the one obtained with the EosY/native byssus. A reasonable explanation is given by the observation of optical images (Fig. 6), which exhibited a selective binding of the MB in cuticle and plaques, which are the most exposed regions of the byssus and the ones richer in DOPA, while under all other conditions the dyes accumulated homogeneously in the diverse byssus regions. These results show that the anionic EosY was more adsorbed from the de-metaled byssus than the metaled one, in which localized positive regions should be present due to the coordinated metallic cations. This may suggest that electrostatic interactions are not the driving force for adsorption and that other interactions may be more prominent. It is important to note that in the experimental conditions used in the adsorbing experiments, the imidazole group of His residue is not protonated. According to literature collagen domains are the binding site for EosY ${ }^{30,31}$ and this may explain the homogeneous adsorption of EosY in the thread also in presence of metals. Dye and byssus aromatic functional groups (e.g. His and DOPA) may also interact through $\pi$-staking. The later require specific geometries according to molecular charge distributions of the aromatic regions. ${ }^{37}$ The EosY electron-rich aromatic structure may interact with His residues, which have an electron-poor aromatic structure, preferentially when His is not coordinating metal ions and has higher mobility (Fig. 7).

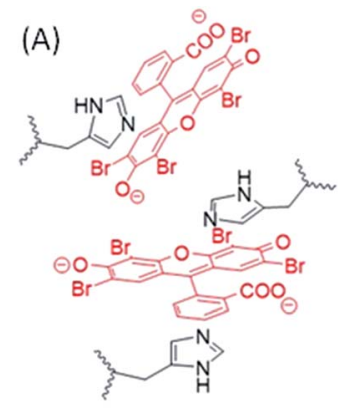

(B)

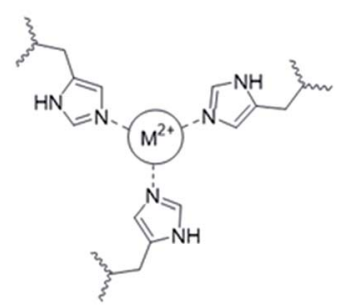

Fig. 7 Schematic representation of a hypothesis of EosY interaction with His into the byssus in the absence of metal ions (A) in the metal coordination site $\mathrm{His}_{3}-\mathrm{M}^{2+}$ of the byssus (B). ${ }^{38}$ The presence of the metal ion reduced the mobility of the imidazole of the His residues and prevents the establishing of $\pi$-staking interactions.

However, this possibility must be further examined. MB uptake from the byssal threads, instead, requires different hypotheses of interaction. No studies have been reported showing MB staining of collagen. Thus, electrostatic and $\pi$-staking interactions should govern the MB uptake by the byssus. The presence of MB into the core - the His rich region - of the metaled byssus suggests that the electrostatic repulsion between metal ion and cationic MB does not affect the uptake. This agrees with the observation that into the de-metaled byssus core, free of localized positive charges, the dye is not up-taken. Indeed, in this condition the MB concentrates into the cuticle, suggesting a repulsion from the core region or a preferential uptake in the cuticle region. We can suppose that also in the case the different efficiencies of the $\pi$-staking interactions, due to the diverse mobility of the imidazole group of His as function of metalation, may control the dye uptake. However, His makes up 2 $\mathrm{mol} \%$ of the total preCol composition (localized at the ends), and it is plausible that the functional groups of other amino acids also play an important role in interacting with dyes. Thus, additional focused experiments will be necessary to understand at molecular level the mechanism of dye uptake by the byssus.

Nonetheless, a strong and tunable absorption effect of byssus as a dye remediation material is demonstrated and this represents the main goal of this research.

Neutral dyes were not used in the current study. Nevertheless, it can be supposed that they can be removed by the byssus. Indeed, in the mechanism proposed, the byssus material is likely to be capable of a number of different interactions besides the electrostatic interaction, including as $\pi$-stacking and hydrophobic interactions that could favor neutral dye removal. Another important point to consider is that the solubility of uncharged dyes in water is likely to be quite low. However, as the byssus is organic and insoluble in most solvents, it could be used as well for the removal of dyes from not water solvents as long as it solvates in them.

\section{Conclusion}

In this work we demonstrated the easy applicability of the byssus as a substrate for dye removal from wastewater of both 
anionic and cationic aromatic dyes. The byssus showed the peculiar ability to switch its affinity for cationic or anionic dyes depending on the presence of metal ions coordinated by its residues. The de-metaled byssus appeared to have a higher affinity for anionic dyes, while the native one binds more efficiently the cationic one. Beside this observation, this research demonstrated that the byssus can absorb in a tunable way up to $10 \mathrm{wt} \%$ of its own weight in dye. In conclusion, the byssus is proposed as a cheap, efficient, and tunable solution to fight industrial dye pollution in water using a biorenewable waste product. The high capability of the byssus to absorb dyes stably is also supported by the parameters obtained by the adsorption isotherm models, offering the perspective to use it as an environmental marker of dye pollution in waters. Moreover, the study of the different interactions between dyes and byssus has provided useful information for byssus future applications in different fields as sensoristics or photonics.

\section{Acknowledgements}

We thank the Consorzio Interuniversitario per la Chimica dei Metalli nei Sistemi Biologici for the support.

\section{Notes and references}

1 K. S. Bharathi and S. T. Ramesh, Appl. Water Sci., 2013, 3, 773-790.

2 S. J. Allen and B. Koumanova, J. Univ. Chem. Technol. Metall., 2003, 40, 175-192.

3 Y. Hu and H. Cheng, Env. Dev., 2013, 8, 57-73.

4 T. Ito, Y. Adachi, Y. Yamanashi and Y. Shimada, Water Res., 2016, 100, 458-465.

5 C. I. Pearcea, J. R. Lloydb and J. T. Guthrie, Dyes Pigm., 2003, 58, 179-196.

6 FAO Yearbook: Fishery and Aquaculture Statistics, Statistics and Information Service of the Fisheries and Aquaculture Department, 2014.

7 N. Vallejos, G. González, E. Troncoso and R. Zuniga, Food Biophys., 2014, 9, 322-331.

8 D. Iribarren, M. T. Moreira and G. Feijoo, Resour., Conserv. Recycl., 2010, 54, 1219-1230.

9 J. Xiong, Y. Qin, E. Islam, M. Yue and W. Wang, Desalination, 2011, 276, 317-321.

10 A. Quintáns-Fondoa, G. Ferreira-Coelhob, R. ParadeloNúñezc, J. C. Nóvoa-Muñozc, M. Arias-Estévezc, M. J. Fernández-Sanjurjoa, E. Álvarez-Rodrígueza and A. Núñez-Delgadoa, J. Cleaner Prod., 2016, 131, 485-490.

11 R. Rezaei, M. Mohadesi and G. R. Moradi, Fuel, 2013, 109, 534-541.

12 S. Hu, Y. Wang and H. Han, Biomass Bioenergy, 2011, 35, 3627-3635.

13 M. I. Jones, H. Barakat and D. A. Patterson, IOP Conf. Ser.: Mater. Sci. Eng., 2011, 18, 192002.

14 F. Byette, C. Pellerin and I. Marcotte, J. Mater. Chem. B, 2014, 2, 6378-6386.
15 F. Byette, A. Laventure, I. Marcotte and C. Pellerin, Biomacromolecules, 2016, 17, 3277-3286.

16 M. J. Harrington and J. H. Waite, Adv. Mater., 2009, 21, 440444.

17 A. Miserez, Y. Li, J. Cagnon, J. C. Weaver and H. J. Waite, Biomacromolecules, 2012, 13, 332-341.

18 E. Vaccaro and J. H. Waite, Biomacromolecules, 2001, 2, 906911.

19 A. Romar-Gasalla, I. M. Rivas-Pérez, R. Paradelo-Núñez, J. C. Nóvoa-Muñoz, M. Arias-Estévez, M. J. FernándezSanjurjo, E. Álvarez-Rodríguez and A. Núñez-Delgado, Geoderma, 2016, 280, 8-13.

20 V. Gupta, K. Suhas, I. Tyagi, S. Agarwal, R. Singh, M. Chaudhary, A. Harit and S. Kushwaha, Global J. Environ. Sci. Manage., 2016, 2, 1-10.

21 C. Duran, D. Ozdes, A. Gundogdu and H. B. Senturk, J. Chem. Eng. Data, 2011, 56, 2136-2147.

22 G. Annadurai, R. S. Juang and D. J. Lee, J. Hazard. Mater., 2002, 92, 263-274.

23 V. Vadivelan and K. V. Kumar, J. Colloid Interface Sci., 2005, 286, 90-100.

24 O. Abdelwahab, A. Nemr, A. E. Sikaily and A. Khaled, J. Aquat. Res., 2005, 31, 1-11.

25 Y. C. Wong, Y. S. Szeto, W. H. Cheung and G. McKay, Process Biochem., 2004, 39, 695-704.

26 M. T. Yagub, T. K. Sen, S. Afroze and H. M. Ang, Adv. Colloid Interface Sci., 2014, 209, 172-184.

27 M. H. Suhre, M. Gertz, C. Steegborn and T. Scheibel, Nat. Commun., 2014, 5, 3392.

28 S. C. T. Nicklisch and J. H. Waite, Biofouling, 2012, 28, 865877.

29 M. J. Harrington, A. Masic, N. Holten-Andersen, J. H. Waite and P. Fratzl, Science, 2010, 328, 216-220.

30 L. Yu, X. Liu, W. Yuan, L. J. Brown and D. Wang, Langmuir, 2015, 31, 6351-6366.

31 J. H. Waite, X. Qin and K. J. Coyne, Matrix Biol., 1998, 17, 93106.

32 T. Hassenam, T. Gutsmann, P. Hansma, J. Sagert and J. H. Waite, Biomacromolecules, 2004, 5, 1351-1355.

33 M. V. Tuttolomondo, L. M. Galdopórpora, L. Trichet, H. Voisin and T. C. M. Federico Desimone, RSC Adv., 2015, 5, 57395-57405.

34 T. Qiang, M. Luo, Q. Bu and X. Wang, Chem. Eng. J., 2012, 197, 343-349.

35 D. L. Callahan, A. J. M. Baker, S. D. Kolev and G. A. Wedd, J. Biol. Inorg Chem., 2006, 11, 2-12.

36 C. N. Z. Schmitt, A. Winter, L. Bertinetti, A. Masic, P. Strauch and M. J. Harrington, J. R. Soc., Interface, 2015, 12, 20150466.

37 C. A. Hunter and J. K. M. Sanders, J. Am. Chem. Soc., 1990, 112, 5525-5534.

38 S. Schmidt, A. Reinecke, F. Wojcik, D. Pussak, L. Hartmann and M. J. Harrington, Biomacromolecules, 2014, 15, 16441652. 